\title{
Protocol
}

\section{Mapping Protein Distributions on Polytene Chromosomes by Immunostaining}

\author{
Renato Paro
}

This protocol was adapted from "Mapping Protein Distributions on Polytene Chromosomes by Immunostaining," Chapter 8, in Drosophila Protocols (eds. Sullivan et al.). Cold Spring Harbor Laboratory Press, Cold Spring Harbor, NY, USA, 2000.

\section{INTRODUCTION}

The formidable size and structure of polytene chromosomes allow mapping of chromosomal protein distributions at very high resolution. This protocol describes the preparation of polytene chromosomes from Drosophila larvae, immunostaining of the chromosomes with a protein of interest, and counterstaining with Giemsa and Hoechst.

\section{RELATED INFORMATION}

Technical modifications incorporated into this protocol that have enabled more reproducible patterns of banding in immunostained chromosomes were initially described by Silver and Elgin (1976). For further details on dissecting larval salivary glands, see Dissection of Larval Salivary Glands and Polytene Chromosome Preparation (Kennison 2008).

\section{MATERIALS}

CAUTIONS AND RECIPES: Please see Appendices for appropriate handling of materials marked with $\langle!\rangle$, and recipes for reagents marked with $<\mathbf{R}>$.

\section{Reagents}

$<!>$ Ammonium sulfate $(50 \%[\mathrm{w} / \mathrm{v}])$ (optional, see Step 23$)$

Antibodies, primary

Affinity-purified primary antibodies are diluted in PBS containing 1\% BSA. The dilutions must be adjusted for each individual primary antibody. Typical dilutions for rabbit polyclonal antibodies range between 1:50 and 1:500. For double-labeling experiments, use primary antibodies raised in two different species.

Antibodies, secondary

Secondary antibodies are diluted in PBS containing 2\% normal serum, obtained from the same species as the secondary antibody. Depending on the method employed, use anti-rabbit IgG $(F c)$ horseradish peroxidase (HRP) conjugate (1:100 dilution), a biotin-conjugated secondary antibody, or a fluorescence-conjugated secondary antibody. For double-labeling experiments, use fluorescence-conjugated secondary antibodies raised to the corresponding type of primary antibody. The dilution factor for each antibody needs to be determined experimentally.

Blocking solution (a small spoonful of nonfat dry milk in $40 \mathrm{~mL} P B S$ )

Bovine serum albumin (BSA)

$<$ ! $>3,3$ '-diaminobenzidine tetrahydrochloride (DAB; Sigma D5637)

Detergent (optional, see Step 2)

Drosophila (see Steps 7-9) 
$<$ !> Entellan (EMD) (optional, see Step 33)

Ethanol (95\%)

$<$ R $>$ Fixing solution

$<$ !>Giemsa (Merck 109204)

Glycerol (99.5\%)

$<!>\mathrm{H}_{2} \mathrm{O}_{2}$ (Merck 107210)

$<\mathrm{R}>$ Hoechst staining solution

$<$ ! $>$ Liquid nitrogen

$<$ !>Methanol (optional, see Step 23)

$<$ R $>$ Mowiol-DABCO stock solution

$<$ R $>$ Nutrient-rich fly medium

$<$ R $>$ Phosphate-buffered saline (PBS; pH 7.5)

$<$ R $>$ Poly-L-lysine solution (0.1\% [w/v] in $\mathrm{H}_{2} \mathrm{O}$; Sigma P8920)

$<$ R $>$ Sodium phosphate buffer $(10 \mathrm{mM}, \mathrm{pH} 6.8)$

$<$ ! $>$ Triton X-100

VECTASTAIN Elite ABC Kit (Vector Laboratories PK 6100) (optional, see Step 30)

$<$ R $>$ Wash solution 1

$<\mathbf{R}>$ Wash solution 2

\section{Equipment}

Aluminum foil

Diamond pen

Eye protection (see Step 21)

Filter paper (e.g., Whatman 3MM)

Humid chamber for slide incubation

Latex gloves

Microscope, dissecting

Microscope, phase-contrast

Pencil with eraser end

Rack for holding slides

Razor blade

Shaking platform

Siliconized coverslips (Corning or equivalent; $22 \times 22 \mathrm{~mm}$ )

Slides

Slide jars

Squashing apparatus (optional, see Step 17)

For extended chromosome-spreading sessions, use the custom-made squashing apparatus (Fig. 1).

Tweezers

\section{METHOD}

\section{Preparation of Poly-L-Lysine-Coated Slides}

1. Place $100-200$ slides in racks.

2. (Optional) Wash slides for $2 \mathrm{~h}$ in a strong detergent.

In general, high-quality slides do not require any pretreatment with detergent. However, in some cases, pretreatment with a strong detergent may be necessary to ensure a homogeneous wetting of the surface by the poly-L-lysine solution in Step 5.

3. Wash for $2 \mathrm{~h}$ in running tap water. Rinse twice in $\mathrm{H}_{2} \mathrm{O}$.

4. Dip slides twice in $95 \%$ ethanol. Air-dry.

5. Dip slides into the poly-L-lysine solution.

The solution should wet the glass surface uniformly and stay on the slides. 


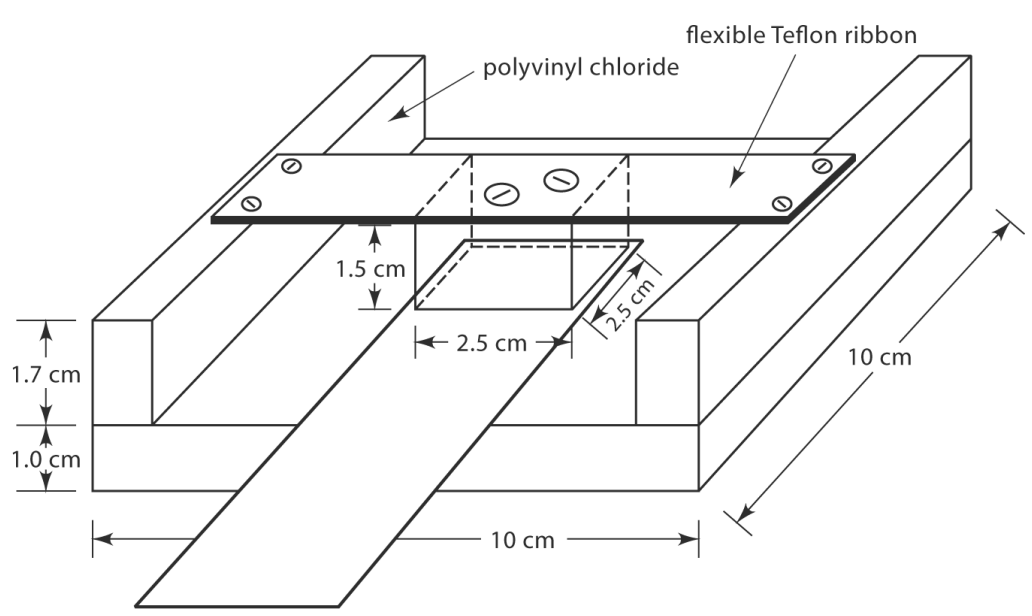

FIGURE 1. Schematic diagram of the squashing apparatus. This apparatus eases the effort when squashing chromosomes over an extended period, and produces more homogeneous pressure over the entire coverslip. A small block made of polyvinyl chloride $(2.5 \times 2.5 \times 1.5 \mathrm{~cm})$ is attached to a flexible Teflon ribbon as shown. The block should hang $\sim 2-3$ $\mathrm{mm}$ above the plane of the holding block (also made of polyvinyl chloride). A piece of filter paper (i.e., Whatman 3MM) and the slide with the coverslip are then positioned on the holding block and held in place by the suspended block.

6. Air-dry the slides, and store them at $4^{\circ} \mathrm{C}$.

\section{Raising Third-Instar Larvae}

The quality of the chromosomes depends critically on the state of nutrition and well-being of the larvae. This is particularly important to consider in cases where the genetic background (e.g., unhealthy mutants or certain transgenic lines) places a heavy toll on the development of the larvae.

7. Raise flies in $175-\mathrm{mL}$ bottles containing nutrient-rich fly medium.

8. Allow the flies to lay eggs just to the point where larvae will hatch under uncrowded conditions (i.e., fewer than 100 larvae in a $175-\mathrm{mL}$ bottle). Let the larvae develop at $18^{\circ} \mathrm{C}$.

9. For salivary gland preparations, use third-instar larvae that are still crawling and have not yet started to pupate.

\section{Dissecting Larval Salivary Glands and Fixing Chromosomes}

10. For each slide, remove two larvae from the bottle, and wash them in PBS.

11. Keep the larvae in PBS, and dissect out the two pairs of salivary glands from each larva using tweezers. Carefully remove most of the attached white fat-body cells without damaging or separating the salivary glands.

For further details on dissecting larval salivary glands, see Dissection of Larval Salivary Glands and Polytene Chromosome Preparation (Kennison 2008).

12. Hold the salivary glands at the common duct with tweezers. Transfer the glands to a drop of fixing solution on a siliconized coverslip.

13. Incubate the glands for $10-20$ min, occasionally stirring with the tip of the tweezers to ensure homogeneous fixation.

The fixation time is an important parameter and needs to be adjusted for every antigen tested. Aim for the shortest time necessary to visualize the signal, as extended fixation times will result in difficulties with spreading the chromosomes. Unlike the sole treatment with acids, e.g., Dissection of Larval Salivary Glands and Polytene Chromosome Preparation (Kennison 2008), the inclusion of formaldehyde in the fixing solution keeps the chromosome sticky, making it more difficult to obtain preparations with well-spread chromosome arms.

\section{Preparing Chromosome Squashes}

14. Take up the coverslip with a poly-L-lysine-treated slide. 
15. Tap the coverslip with the eraser end of a pencil until the cells are broken up. This can be monitored best when done against a black background.

16. Move the eraser end of the pencil over the coverslip to spread the chromosomes. To avoid extensive movement of the coverslip, hold down the coverslip with the tip of a finger (use latex gloves to prevent acid burns). Do not continue to spread the specimen if the coverslip sticks to the slide, as this will result in shearing of the chromosomes.

The chromosomes are very brittle at this point of the process. The extent of spreading depends on the constitution and size of the chromosomes, as well as on the time of fixation.

17. Invert the slide, and place it over one layer of filter paper. Apply firm pressure with the thumb; the chromosomes become flattened and pressed to the slide.

The squashing apparatus (Fig. 1) can substantially ease this step because it allows more firm and homogeneous pressure on the chromosomes without the risk of moving the coverslip.

18. Examine the preparation by phase-contrast microscopy. Use only preparations with well-spread chromosome arms showing high-contrast banding patterns.

19. Mark the position of the coverslip with a diamond pen.

20. Freeze the slide in liquid nitrogen.

21. Remove coverslip with a razor blade (wear appropriate eye protection).

22. Immediately dip the slide into PBS, and wash it for $15 \mathrm{~min}$ with gentle shaking. Repeat this wash once in fresh PBS.

23. Proceed with the immunostaining as described below. Alternatively, keep the slide (for up to $1 \mathrm{wk}$ ) in $100 \%$ methanol or in $50 \%(\mathrm{w} / \mathrm{v})$ ammonium sulfate at $4{ }^{\circ} \mathrm{C}$.

Certain antigens might be affected by exposure to methanol.

\section{Immunostaining}

24. Wash stored slides twice in PBS (10 min each wash).

25. Wash slides once in PBS containing $1 \%$ Triton $\mathrm{X}-100$ for $10 \mathrm{~min}$.

26. Incubate slides in a slide jar containing blocking solution for $1 \mathrm{~h}$ at room temperature or overnight at $4^{\circ} \mathrm{C}$ with gentle shaking. Drain off excess solution.

27. Add $40 \mu \mathrm{L}$ of diluted affinity-purified primary antibody to each slide, and cover with a coverslip. Incubate the slides for $1 \mathrm{~h}$ at room temperature.

28. Incubate the slides overnight at $4^{\circ} \mathrm{C}$ in a humid chamber.

29. Rinse the slides in PBS to remove the coverslips, and place them in a rack. Wash the slides three times in blocking solution (5 min each wash) with thorough shaking.

30. Probe the slides with a secondary antibody detection system using one of the three methods below. If only weak signals are observed using enzyme-coupled secondary antibodies (Steps 30.i-30.vi), amplification with the biotin-avidin system might be helpful (Steps 30.vii-30.xiii). Secondary antibodies tagged with a fluorochrome (Steps 30.xiv-30.xvii) can be used to speed up the detection procedure, as well as for double-labeling experiments.

\section{To immunostain using enzyme-coupled secondary antibodies:}

i. Rinse slides in PBS and remove excess solution.

ii. Add $40 \mu \mathrm{L}$ of secondary antibody (e.g., anti-rabbit IgG [Fc] HRP conjugate, 1:100 dilution) to each slide, and cover with a coverslip. Incubate for $1 \mathrm{~h}$ at room temperature in a humid chamber.

Often, commercially available secondary antibody preparations show a high affinity for Drosophila nuclear proteins, resulting in some excellent signals on the chromosomes. As such, a corresponding control experiment should always be included.

iii. Rinse slides in PBS and place in rack. Wash slides in wash solution 1 for 15 min and then in wash solution 2 for $15 \mathrm{~min}$. Shake rack thoroughly during the washing procedure. 
iv. Rinse slides in PBS. Add $100 \mu \mathrm{L}$ of a solution containing $0.5 \mathrm{mg} / \mathrm{mL} \mathrm{DAB}$ and $0.01 \% \mathrm{H}_{2} \mathrm{O}_{2}$.

v. To prevent overstaining, follow the appearance of the signals under the microscope using bright-field optics. Stop the reaction by dipping the slides in PBS. Wash in PBS for 10 min.

vi. Counterstain with Giemsa (see Steps 30-33).

To immunostain using biotin-conjugated secondary antibodies and enzymatic detection with the avidin/biotin system:

vii. To each slide, add $40 \mu \mathrm{L}$ of secondary antibody conjugated to biotin, and cover with a coverslip. Incubate for $1 \mathrm{~h}$ at room temperature in a humid chamber.

viii.During secondary antibody incubation, mix the biotin solution and the avidin-HRP solution to allow the formation of complexes. Mix $40 \mu \mathrm{L}$ of solution $A$ and $40 \mu \mathrm{L}$ of solution $B$ (from the VECTASTAIN Elite ABC kit) in $1 \mathrm{~mL}$ of PBS containing $0.1 \%$ BSA for $10 \mathrm{~min}$ at room temperature.

ix. After incubation with secondary antibodies, rinse slides twice in PBS containing $0.1 \%$ BSA (10 min each). Remove excess liquid.

x. Place $50 \mu \mathrm{L}$ of the biotin-avidin-HRP mix on the preparation and cover with a coverslip. Incubate the slides for $40 \mathrm{~min}$ at room temperature in a humid chamber.

xi. Wash slides in wash solution 1 for $15 \mathrm{~min}$ and then in wash solution 2 for $15 \mathrm{~min}$.

xii. Rinse slides in PBS. Add $100 \mu \mathrm{L}$ of a solution containing $0.5 \mathrm{mg} / \mathrm{mL} \mathrm{DAB}$ and $0.01 \% \mathrm{H}_{2} \mathrm{O}_{2}$.

xiii. To prevent overstaining, follow the appearance of the signals under the microscope using bright-field optics. Stop the reaction by dipping slides in PBS. Wash in PBS for $10 \mathrm{~min}$.

\section{To immunostain using fluorescence-coupled secondary antibodies:}

xiv. Rinse slides in PBS and remove excess solution.

After this point, perform all steps under dimmed-light conditions. During incubations, wrap the slide jars in aluminum foil to minimize bleaching of fluorochromes.

xv. Add $40 \mu \mathrm{L}$ of diluted fluorescence-conjugated secondary antibody solution to the slide, and cover with a coverslip. Incubate for $1 \mathrm{~h}$ at room temperature in a humid chamber.

For double-labeling experiments, both secondary antibodies of the appropriate type can be applied simultaneously during this step.

xvi. Rinse slides in PBS and place in a rack. Wash slides in wash solution 1 for 15 min and then in wash solution 2 for $15 \mathrm{~min}$.

xvii. Store slides in PBS at $4^{\circ} \mathrm{C}$ in the dark or proceed to counterstain with Hoechst 33258 (see Steps 35-37).

\section{Giemsa Staining}

Chromosomes with HRP signals should be counterstained with Giemsa.

31. Prepare a 1:130 dilution of Giemsa in $10 \mathrm{mM}$ sodium phosphate buffer ( $\mathrm{pH} 6.8)$.

32. Stain slides for $30 \mathrm{sec}$ to $1 \mathrm{~min}$. Rinse slides by dipping several times into $\mathrm{H}_{2} \mathrm{O}$.

33. Mount in $99.5 \%$ glycerol with a coverslip. Immediately examine the slides under the microscope. Giemsa stain will fade within a few hours. However, chromosomes can be washed in PBS and restained.

34. For storage, slides can be frozen at $-20^{\circ} \mathrm{C}$. Entellan (EMD) can be used as a permanent mounting solution.

Staining with Hoechst 33258

Chromosomes with fluorescent signals can be counterstained with Hoechst 33258. However, banding patterns produced by the fluorescent DNA stains are often difficult to compare with the published polytene chromosome-banding patterns. It is often more convenient to simply use phase-contrast to correlate the fluorescent signal with the corresponding chromosome bands. 


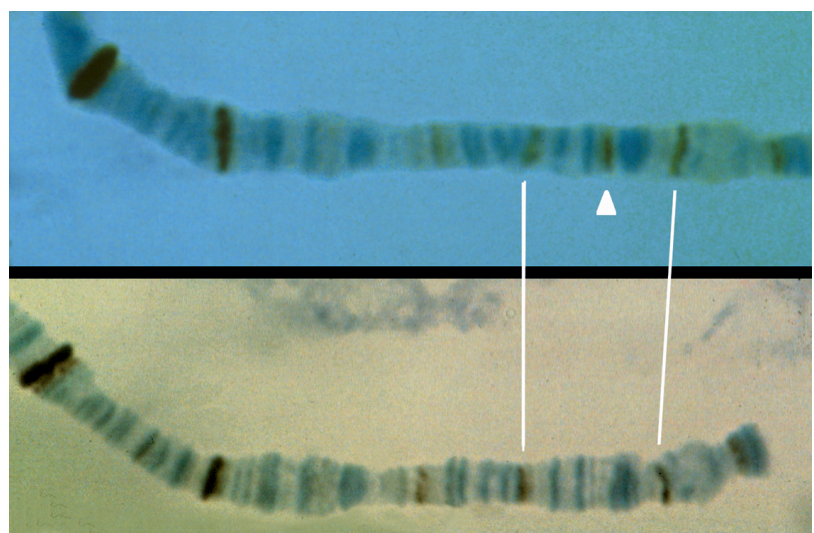

FIGURE 2. Distribution of the Polycomb protein at section 43-49 of the second chromosome visualized with enzymeconjugated (HRP) secondary antibodies. (Top) Chromosome with a transgene construct containing a Polycomb-binding site (PRE) at 44E (arrowhead); (bottom) wild-type chromosome. (For color figure, see doi: 10.1101/pdb.prot4995 online at www.cshprotocols.org.)

35. Stain slides in Hoechst staining solution for 5 min.

36. Mount preparations with fluorescent signals in Mowiol-DABCO stock solution.

37. Examine the slides using a fluorescence microscope.

\section{TROUBLESHOOTING}

Problem: Background staining is high.

[Steps 30, 33, or 37]

Solution: The concentration of $\mathrm{NaCl}$ in wash solution 2 can be raised to $500 \mathrm{mM}$.

Problem: HRP staining is weak.

[Step 30]

Solution: Silver treatment of the preparation turns the brown color of a DAB signal to black, thus substantially improving the contrast, which is very convenient when black-and-white photography is used for documentation. Use the Sigma FAST DAB with metal enhancer (Sigma D0426), following the supplier's instructions, but shorten the metal amplification step to $1 \mathrm{~min}$.

Problem: In Giemsa-stained slides, staining of chromosome bands appears too weak under brightfield optics.

[Step 33]

Solution: Repeat Giemsa staining starting with Step 32.

\section{DISCUSSION}

Immunostaining of polytene chromosomes is most often used for mapping distributions of chromosome-associated proteins, identifying and characterizing cis-regulatory DNA elements bound by particular proteins, and mapping functional protein domains necessary for chromosomal binding or other activities, such as the interaction with other partner proteins. For example, a transgene construct containing a cis-regulatory DNA element may create a new protein-binding site at the integration site on a chromosome (Fig. 2).

\section{REFERENCES}

Kennison, J.A. 2008. Dissection of larval salivary glands and polytene chromosome preparation. CSH Protocols (this issue) doi: $10.1101 /$ pdb.prot4708
Silver, L.M. and Elgin, S.C.R. 1976. A method for determination of the in situ distribution of chromosomal proteins. Proc. Natl. Acad. Sci. 73: 423-427. 


\section{Mapping Protein Distributions on Polytene Chromosomes by Immunostaining}

Renato Paro

Cold Spring Harb Protoc; doi: 10.1101/pdb.prot4714

\begin{tabular}{|c|c|}
\hline $\begin{array}{r}\text { Email Alerting } \\
\text { Service }\end{array}$ & Receive free email alerts when new articles cite this article - click here. \\
\hline $\begin{array}{l}\text { Subject } \\
\text { Categories }\end{array}$ & $\begin{array}{l}\text { Browse articles on similar topics from Cold Spring Harbor Protocols. } \\
\text { Antibodies, general (289 articles) } \\
\text { Cell Biology, general (1382 articles) } \\
\text { Cell Imaging (525 articles) } \\
\text { DNA Modification/Epigenetics (42 articles) } \\
\text { DNA:Protein Interactions ( } 74 \text { articles) } \\
\text { Drosophila (272 articles) } \\
\text { Imaging/Microscopy, general (579 articles) } \\
\text { Immunostaining (139 articles) } \\
\text { Laboratory Organisms, general (923 articles) } \\
\text { Molecular Biology, general (1293 articles) } \\
\text { Proteins and Proteomics, general (575 articles) } \\
\text { Visualization (524 articles) } \\
\text { Visualization of Proteins (107 articles) }\end{array}$ \\
\hline
\end{tabular}

